\title{
Stigma after being newly diagnosed with acute leukemia: the contributing factors among those patients
}

Miaoran Lin

Fujian Medical University

Rong Hu ( $\nabla$ ronghu1246@fjmu.edu.cn )

Fujian Medical University https://orcid.org/0000-0002-3039-0498

Xiao-Ying Jiang

Fujian Medical University

Ying-chun Lin

First Affiliated Hospital of Fujian Medical University

Yi-mei Weng

Fujian Medical University Union Hospital

\section{Research Article}

Keywords: Acute leukemia, Stigma, Coping, Anxiety, Depression, Cancer

Posted Date: October 6th, 2021

DOI: https://doi.org/10.21203/rs.3.rs-869928/v1

License: (a) (i) This work is licensed under a Creative Commons Attribution 4.0 International License. Read Full License 


\section{Abstract \\ Objective:}

To date, little research has been done to answer whether people with acute leukemia (AL) experience stigma. No previous studies investigated stigma and its relationship with most common negative emotion and coping styles in patients newly diagnosed as AL. Therefore, the study was designed to find out stigma and its possible contributing factors among the patients with initial diagnosis of AL.

\section{Methods:}

A total of 167 patients newly diagnosed as AL were recruited and investigated by Social Impact Scale (SIS), Medical Coping Modes Questionnaire, Hospital Anxiety and Depression Scale. The data was analyzed by multivariate linear regression to identify influencing factors of stigma.

\section{Results:}

The mean score of SIS was $60.61 \pm 9.45$, with $13.17 \%$ of patients at high level. Almost individuals experienced anxiety or depression during first chemotherapy after diagnosis. The main coping strategy was acceptance-resignation. Patients with following characteristics tend to experienced stronger stigma: male, childless, lower family income, higher degree of dependence, having chronic diseases, more severe depression, and tendency to adopt acceptance-resignation.

\section{Conclusions:}

Patients newly diagnosed as AL were in moderate level of stigma. Effective measures were suggested to alleviate stigma the patients undergoing when struggling for complete remission, especially for those exposed to risk factors of stigma.

\section{Introduction}

Acute leukemia $(A L)$ is a dreaded and life-threatening illness characterized by acute onset, rapid progression and poor prognosis[1]. A recent survey of Global Cancer Statistics in 185 countries has found that 437,033 new cases and 309,006 deaths of leukemia occurred worldwide in 2018[2], on a general increase, including China[3]. Although chemotherapy remains the primary treatment for AL and offers great potential for induced remission rate, patients, especially newly diagnosed with AL, suffer a lot not only from a range of physical function impairments such as fatigue, pain, nausea and vomiting[4], but also a series of psychological disorders like anxiety, desperation, self-image disorder and low self-esteem as well[5-7]. 
It has long been recognized that various types of illnesses, including hematologic malignancy, are associated with stigma[8, 9], which now being considered as a pervasive fundamental cause of population health inequalities[10]. Stigma and its concept were first mentioned in 1963 by sociologist Goffman[8]. It was described as an attribute which is deeply discrediting due to the blemishes of body image, unpleasant stereotypes individual having, or being a member of an undesirable social group. In 2000 , Fife put forward a concept in analysis of the relationship between mechanisms of stigma and the self (self-esteem, body image, and mastery)[11]. In his study, the conceptualization of stigma is grounded in Modified Labeling Theory. Based on this basis, he constructed the Social Impact Scale (SIS) containing four dimensions named social rejection, social isolation, financial insecurity, and internalized shame as a tool to assess the cancer-related stigma. It is generally believed that public stigma is related to social discrimination and patients' negative self-perception, which can be internalized into internal stigma[12] and lead to proximal or distal consequences such as delay in seeking medical treatment[13], decline in treatment adherence[14], poor health outcomes, reduced chance for survival, lower QOL or even abandoning therapy in desperation.

When people diagnosed with $\mathrm{AL}$, they already developed with various functional impairments due to anemia, infection, or hemorrhage trend. The severity of the illness, and the decrease in functional healthy status, did result in patients unable to participate in normal social life and setting them apart from others[5]. Consequently, the more social isolated they involving, the more social rejected they may feel. Besides, the cost of AL treatment is enormous, some chemotherapy drugs are not covered by the basic medical insurance system in China. This could evidently bring grievous socioeconomic pressures for most patients, especially disadvantaged groups labeled as drain on economy or having urgent demand for social welfare in very beginning[15], putting them in particular risk for low self-esteem[6].

What's more, in China, people seldom talk about deaths for it was ominous. Even after confirmation of AL diagnosis, the desire to maintain normality in life can sometimes make them go to extreme lengths to conceal self-image disorder from others. Some social taboos frequently prevent individuals from seeking conventional health-care help, especially for socially stigmatized diseases such as cancer[16]. For those survivors, they concern about the disclosure of their medical history, making them feel more vulnerable to the "internalized shame", thus leading to the occurrence of stigma. Given the circumstances and characteristics of AL, we thereby use SIS to assess the level of stigma among patients in our study.

Numerous studies $[5,17]$ have demonstrated anxiety and depression are two most common mental health disorders and negatively affect clinical outcomes in patients with AL. Regardless of highly developed medical science and increased survivorship, when people newly diagnosed with incurable disease, they experience with co-morbid depression or anxiety, and it was significantly associated with stigma[18]. Those with high level of depression or anxiety are predisposed to developed negative attitudes toward cancer, to hold stereotypical views of themselves and to specially reinforce the feelings of stigma[19, 20]. Such circumstance can also be found in AL patients during our clinical daily work. So, we wonder and want to identify if these two very common negative emotions contribute to stigma in patients newly diagnosed as AL. 
Coping represents the strategies that individuals use to manage the internal and external demands and plays a crucial role in people instinctively reacting to life stressors, threats, or specific situation they encounter[21]. After diagnosed as AL, they may experience a variety of practical, social, emotional, and existential concerns due to the disease, side effects of treatments, and poor prognosis[22]. As to AL patients, it's quite clear that positive coping is associated with higher QOL, reduced depressive symptoms and better clinical outcomes[23], while negative coping responses were found to be associated with greater stigma[24, 25]. When people adopt positive coping skills, like being active in seeking for solutions or feeling confident in their coping strategies, stigma decreases[26]. Therefore, we aim to find out whether coping strategy, the important variable, is related to stigma in AL patients, and can be considered as protective factor as other studies have revealed.

The occurrence of cancer stigmatization among survivors ranges from $13 \%-80 \%[20,27,28]$. However, little research has been designed to identify whether people with AL experience stigma. Furthermore, no previous studies investigated the prevalence of stigma and its relationship with most common negative emotions and significant coping styles in patients newly diagnosed as AL. Based on the hypothesis that stigma experiencing may differ from different cultures and cancer types, therefore, the study was conducted to address the gap in understanding of stigma and its possible influencing factors among patients with first diagnosis of AL in China.

\section{Methods}

\subsection{Study design and participants}

A cross-sectional study was conducted using convenience sampling method in 2 tertiary hospitals of Fujian from August 2017 to May 2019. Hospitalized patients eligible for this study met the inclusion criteria: 1)a diagnosis of AL by clinical bone marrow aspiration for first time; $2 \square$ age $\geq 18$ years $₫ 3 \rrbracket a b l e$ to read or speak mandarin $₫ 4 \rrbracket$ willing to be enrolled in this study. Participants with mental illness, cognitive disorders, AIDS, other malignances or being not informed of illness after diagnosis were excluded. Before the survey, participates were provided written informed consent and were informed the procedure of the study in detail. Confidentiality was assured in all data for stigma is a sensitive topic. Participates completed the questionnaires by themselves except when they were in doubt or requested standard explanations. All questionnaires filled out were checked and kept in time by researchers.

\subsection{Measurements}

\subsubsection{Demographic \& Clinical questionnaire}

Demographic information (including gender, age, et al.) and clinical data (eg, type of leukemia, hospital stays) were recorded in Table 1. 
Table 1

Demographic \& clinical data of the patients and the comparison of stigma among them

\section{Characteristic}

Gender

Male

Female

Age(years)

$18-44$

$45-59$

$\geq 60$

Marital status

Married

Others (eg. unmarried or divorce or widowed)

Fertility circumstance

Child

Childless

Educational level

Less than junior high school

High school/technical secondary school

Junior college

Bachelor degree or higher

Family per capita monthly income (yuan RMB)

Q1000

1000-1999

2000-2999

3000-3999

4000-4999

$\geq 5000$ $\mathrm{n}(\%)$ Stigma Mean \pm SD

$t / F(p)$

$2.305\left(.022^{\star}\right)$

$90(53.9 \%) \quad 62.26 \pm 8.65$

$77(46.1 \%) \quad 59.07 \pm 9.21$

$5.551\left(.005^{\star}\right)$

$76(45.5 \%) \quad 63.04 \pm 8.96$

$55(32.9 \%) \quad 57.67 \pm 9.56$

$36(21.6 \%) \quad 59.94 \pm 9.13$

$-2.254\left(.026^{\star}\right)$

$133(79.6 \%) \quad 59.78 \pm 9.13$

$34(20.4 \%) \quad 63.82 \pm 10.08$

$-4.045$

$\left(.000^{\star \star}\right)$

$141(84.4 \%) \quad 59.39 \pm 9.236$

$26(15.6 \%) \quad 67.19 \pm 7.83$

$2.133(.098)$

$78(46.7 \%) \quad 60.99 \pm 8.25$

$43(25.7 \%) \quad 60.49 \pm 11.85$

$34(20.4 \%) \quad 58.21 \pm 9.03$

$12(7.2 \%) \quad 65.33 \pm 6.72$

$-3.721\left(.003^{\star}\right)$

$36(21.6 \%) \quad 67.33 \pm 7.89$

$53(31.7 \%) \quad 65.40 \pm 7.85$

45(26.9) $\quad 62.53 \pm 9.54$

$20(12.0 \%) \quad 57.58 \pm 8.29$

$9(5.4 \%) \quad 59.98 \pm 9.51$

$4(2.4 \%) \quad 60.00 \pm 12.78$ 


\begin{tabular}{|c|c|c|c|}
\hline Characteristic & $\mathrm{n}(\%)$ & $\begin{array}{l}\text { Stigma Mean } \pm \\
\text { SD }\end{array}$ & $t / F(p)$ \\
\hline Place of residence & & & $0.271(.763)$ \\
\hline Rural or village & $50(29.9 \%)$ & $61.14 \pm 9.78$ & \\
\hline Suburban (including town, county) & $63(37.7 \%)$ & $59.92 \pm 9.574$ & \\
\hline Urban & $54(32.3 \%)$ & $60.91 \pm 9.10$ & \\
\hline Medical insurance & & & $1.071(.373)$ \\
\hline $\begin{array}{l}\text { New agricultural cooperative medical } \\
\text { insurance }\end{array}$ & $88(52.7 \%)$ & $59.33 \pm 8.75$ & \\
\hline Urban Resident Basic Medical insurance & $56(33.5 \%)$ & $62.43 \pm 9.37$ & \\
\hline Urban employee basic medical insurance & 15(9.0\%) & $61.53 \pm 10.29$ & \\
\hline Commercial insurance & $5(3.0 \%)$ & $61.80 \pm 14.03$ & \\
\hline Self-paid (uninsured) & $3(1.8 \%)$ & $57.33 \pm 18.34$ & \\
\hline Employment status & & & $1.901(.097)$ \\
\hline Full-time employment & $56(33.5 \%)$ & $59.95 \pm 10.46$ & \\
\hline Part-time employment & $25(15.0 \%)$ & $60.72 \pm 9.27$ & \\
\hline Freelance/Self-Employed & $35(21.0 \%)$ & $58.74 \pm 8.02$ & \\
\hline On sick leave & $21(12.6 \%)$ & $60.67 \pm 9.85$ & \\
\hline Retired & $16(9.6 \%)$ & $60.63 \pm 9.58$ & \\
\hline Student & $14(8.4 \%)$ & $67.57 \pm 5.53$ & \\
\hline Type of leukemia & & & $-1.009(.314)$ \\
\hline Acute myelogenous leukemia & $62(37.1 \%)$ & $59.65 \pm 9.95$ & \\
\hline Acute lymphocytic leukemia & $105(62.9 \%)$ & $61.17 \pm 9.14$ & \\
\hline Notification of serious or critical illness & & & $-0.326(.745)$ \\
\hline Yes & $112(67.1 \%)$ & $60.44 \pm 9.46$ & \\
\hline No & $55(32.9 \%)$ & $60.95 \pm 9.49$ & \\
\hline \multicolumn{3}{|c|}{ Degree of disease dependence (Barthel Index, points) } & $2.592(.039 *)$ \\
\hline Independent (100) & $14(7.8 \%)$ & $56.86 \pm 6.31$ & \\
\hline Mildly dependent (75-95) & $49(32.3 \%)$ & $62.22 \pm 7.04$ & \\
\hline
\end{tabular}




\begin{tabular}{|llll|}
\hline Characteristic & $\mathrm{n}(\%)$ & $\begin{array}{l}\text { Stigma Mean } \pm \\
\text { SD }\end{array}$ & t/F(p) \\
\hline Moderately dependent $(50-70)$ & $63(37.1 \%)$ & $60.56 \pm 8.91$ & \\
\hline Severely dependent $(25-45)$ & $37(20.4 \%)$ & $62.76 \pm 9.43$ & \\
\hline Utterly dependent $(0-20)$ & $4(2.4 \%)$ & $69.75 \pm 6.50$ & \\
\hline Chronic diseases & & & $-0.212\left(.036^{*}\right)$ \\
\hline Yes & $48(28.7 \%)$ & $63.60 \pm 9.66$ & \\
\hline No & $119(71.3 \%)$ & $60.57 \pm 7.79$ & \\
\hline Hospital stays (days) & & & \\
\hline$\leq 10$ & $46(27.5 \%)$ & $59.52 \pm 9.39$ & \\
\hline $11 \sim 19$ & $20(12.0 \%)$ & $63.10 \pm 11.16$ & \\
\hline$\geq 20$ & $64(38.3 \%)$ & $60.25 \pm 9.92$ & \\
\hline$\geq 30$ & $37(22.2 \%)$ & $61.22 \pm 7.60$ & \\
\hline$* P \otimes 0.05 * * P \otimes 0.01$ & & & \\
\hline
\end{tabular}

\subsubsection{Social Impact Scale (SIS)}

The 24-item SIS, developed by Fife[11] in 2000, was translated into Chinese by Pan[29] in 2007. The scale consists of 4 domains: internalized shame ( 5 items), social isolation (7 items), social rejection (9 items), and financial insecurity ( 3 items). The response of items is rated on 4-point Likert scale ( $1=$ extremely agree to $4=$ completely disagree). A higher average item score indicates a greater level of perceived stigma: high (3 4), moderate (2-2.99), or low (1-1.99)[11]. The Cronbach's coefficient in this study was 0.872 .

\subsubsection{Hospital Anxiety and Depression Scale (HADS)}

The HADS, designed by Snaith, was introduced into China in 1983[30]. It has a good rapid screening efficacy and is widely used among cancer patients in hospitals. The scale contains 2 subscales (HAS, HDS), 14 items, adopting 4-point Likert scale $(0=$ inexistence to $3=$ frequently exist). The total scores of each subscale ranges from 0 to 21 ( 0 to 7,8 to 10 and 11 to 20 indicates asymptomatic, suspicious and positive respectively). In this study, we choose 10 as the optimal critical value. The Cronbach's coefficient in this study was 0.892 .

\subsubsection{Medical Coping Modes Questionnaire (MCMQ)}

The MCMQ, established by Feifel in 1897 and revised into Chinese version by Shen[31]. It's composed of 20 items covering 3 subscales: confrontation, avoidance, and acceptance-resignation. Each item is rated on 4-point Likert scale (1 to 4), the higher score of the subscale is, the more individual tends to adopt that 
kind of coping strategy. The Cronbach's coefficient of Chinese version in 3 subscales were $0.69,0.60$, and 0.76 respectively. In this study, the Cronbach's coefficient was $0.721,0.718$, and 0.746 .

\subsection{Statistical methods}

The data was stored in Excel and analyzed by SPSS 22.0. The continuous variables and categorical variables were described by range (mean \pm standard deviation) and frequencies (percentages) respectively. We compared stigma among patients with different baseline characteristics by using independent samples $t$ tests for 2-level variables or 1-way ANOVA for variables with 3 or more levels. Pearson's correlation was analyzed to demonstrate the correlations between stigma and anxiety, depression, coping strategies. We put variables from univariate analysis and Pearson's correlation above into multiple linear regressions analysis to determine the statistically significant factors that contributing to stigma. An alpha level of 0.05 was selected as criterion for statistical significance.

\section{Results}

\subsection{Sample characteristics}

A total of 179 patients eligible for inclusion criteria were recruited into our study. 12 of them dropped out because of weakness and fatigue ( 5 patients), depression from sensitive content of questionnaires (4 patients) and other unspecified reasons (3 patients). Finally, 167(93.30\%) patients aged $18 \sim 78$ (mean = $45.34, S D=16.30)$ completed the questionnaires. There was no statistically significant difference between responders and non-responders in distribution of baseline data. Table 1 presents the demographic \& clinical data of the patients and the comparison of stigma among those with different characteristics.

\subsection{Stigma of the patients newly diagnosed as $\mathrm{AL}$}

The score of SIS ranges from 38 to 81 (mean $=60.61, S D=9.45$ ), in moderate level of stigma. Approximately 22(13.17\%) of them were at high while 129(77.25\%) were at moderate. The order of subscales from top to low based on average score of items were financial insecurity, internalized shame, social rejection, and social isolation (Table 2). 
Table 2

Stigma among patients newly diagnosed as $\mathrm{AL}$

\begin{tabular}{|llllll|}
\hline Variables & Scale score & Item score & $\mathbf{N}(\%)$ & & \\
\cline { 5 - 6 } & Mean \pm SD & Mean \pm SD & Low level & Moderate level & High level \\
\hline Stigma & $60.61 \pm 9.45$ & $2.53 \pm 0.39$ & $16(9.58 \%)$ & $129(77.25 \%)$ & $22(13.17 \%)$ \\
\hline Financial insecurity & $8.37 \pm 2.12$ & $2.79 \pm 0.71$ & $18(10.77 \%)$ & $64(38.32 \%)$ & $85(50.90 \%)$ \\
\hline Internalized shame & $13.35 \pm 3.40$ & $2.67 \pm 0.68$ & $26(15.57 \%)$ & $72(43.11 \%)$ & $69(41.32 \%)$ \\
\hline Social rejection & $22.29 \pm 5.81$ & $2.48 \pm 0.65$ & $34(20.36 \%)$ & $92(55.09 \%)$ & $41(24.55 \%)$ \\
\hline Social isolation & $16.60 \pm 5.25$ & $2.37 \pm 0.75$ & $57(34.13 \%)$ & $66(39.52 \%)$ & $44(26.35 \%)$ \\
\hline
\end{tabular}

\subsection{The correlations between stigma and anxiety, depression, coping strategies}

The mean scores of HADS, HAS, HDS were 26.67 (SD = 4.31), $13.30(\mathrm{SD}=2.79), 13.37(\mathrm{SD}=2.62)$ respectively. Both of anxiety $(r=0.355 \mathrm{p} \otimes 0.001)$ and depression $(r=0.440 \mathrm{p} \otimes 0.001)$ were positively associated with stigma. Besides, the mean sores of confrontation, avoidance and acceptance-resignation coping strategies were $17.74(S D=4.99), 15.56(S D=3.67)$ and $13.40(S D=3.23)$ respectively. The results

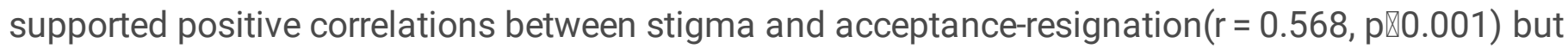
remarkably negative in confrontation ( $r=-0.546, \mathrm{p} \otimes 0.001)$ and avoidance $(r=-0.441, \mathrm{p} \otimes 0.001)$.

\subsection{Influencing factors of stigma in patients newly diagnosed with AL}

Multiple regression analysis was conducted with stigma score as the dependent variable, while demographic \& clinical data, anxiety, depression, and coping strategies as the independent variables. The correlations of these predicting variables were $0.001 \sim 0.588$, in a moderate degree of correlation. In the collinearity diagnosis, the tolerance value $(\mathrm{TOL})$ of independent variables was $0.374 \sim 0.876(\otimes 0.1)$, the variance expansion coefficient (VIF) was 1.142-2.765, all of which were below 3, less than 10 of the evaluation index, which means the problem of multiple collinearity among independent variables involved in the regression analysis was not obvious. The results showed that 9 variables were entered into the regression equation after multiple regression analysis. Among them, the greatest contributor was acceptance-resignation, followed by confrontation (Table 3). 
Table 3

Influencing factors of stigma in patients newly diagnosed as $\mathrm{AL}$

\begin{tabular}{|c|c|c|c|c|}
\hline Variables & B & $\boldsymbol{\beta}$ & $\mathrm{t}$ & $\mathbf{P}$ \\
\hline Constant & 52.031 & & 6.283 & 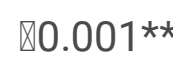 \\
\hline Acceptance-resignation (MCMQ) & 0.825 & 0.282 & 3.936 & $\varangle 0.001^{\star \star}$ \\
\hline Fertility circumstance & 6.809 & 0.262 & 2.963 & $0.004^{\star}$ \\
\hline Confrontation (MCMQ) & -4.024 & -0.190 & -3.013 & $0.003^{*}$ \\
\hline Combined with chronic diseases & -.341 & -0.180 & -2.328 & $0.021^{\star}$ \\
\hline Depression & 1.711 & 0.171 & 2.575 & $0.011^{\star}$ \\
\hline Family per capita monthly income(yuan RMB) & -1.298 & 0.170 & -2.968 & $0.004^{\star}$ \\
\hline Avoidance (MCMQ) & -0.411 & -0.160 & -2.285 & $0.024^{\star}$ \\
\hline Degree of disease dependence & 0.512 & 0.143 & 2.270 & $0.025^{\star}$ \\
\hline Gender & -2.681 & -0.142 & -2.469 & $0.015^{\star}$ \\
\hline
\end{tabular}

\section{Discussions}

\subsection{The level of stigma among patients with initial diagnosis of $\mathrm{AL}$}

Patients with initial diagnosis of AL have moderate level of stigma, which is consistent with most relevant studies[11, 24,25] that using SIS to assess stigma of HIV or cancer. As compared to latest studies of them, AL patients scored higher than lung cancer[13] and prostate cancer[14], but lower than HIV [11], colorectal cancer with stoma[12] and breast cancer[14]. The potential underlying reason for the difference may largely due to the type of cancer. The more serious self-image disorder of the disease the patients expose, the more stigma they might trend to experience. Of four dimensions of SIS, Chinese AL patients scores highest in financial insecurity, followed by internalized stigma. Financial insecurity is a specific consequence of discrimination related to emerging financial crisis individual facing[11]. Pricivel et al[32] put forward a conceptual framework of "financial toxicity" illustrating financial burden and distress of patients with cancer is nowhere. The latter, in general, are combined with anxiety, depressed and self-blaming. Compared to other types of cancers, financial insecurity in AL patients seems more pressing, particularly in initial treatment period[32]. Besides, since AL is frequently recognized as one of most panic diseases, patients with $\mathrm{AL}$ are thought to be unproductive and too fragile to continue the work[13]. From the observation of our routine work, they do concern a lot about how much treatment was going to cost and whether the family was able to afford? Those who have financial insecurity were overly 
concerned about being discriminated and more vulnerable to hold self-accusations of themselves, especially when they have to sale assets or borrow money during induced remission stage. Suggesting clinical care providers should pay attention to the patients who having financial insecurity.

\subsection{The influencing factors of the stigma 4.2.1 Medical coping strategies}

Coping strategy has a significant predictive effect on stigma, and reducing maladaptive coping may largely alleviate experienced stigma[33]. In this study, patients with new diagnosis of AL tend to adopt acceptance-resignation but no confrontation as coping strategy in dealing with their illness and they scored higher than AL patients reported before[34]. Our results indicates that coping strategies are influencing factors of stigma with acceptance-resignation having strongest impact efficiency. Individuals coping with disease via acceptance-resignation experienced stronger stigma while it's opposite in confrontation. Use of acceptance resignation as a coping strategy was forcefully manifest in lifethreatened patients with little expectation of recovery and appeared to be negatively linked to effectiveness of coping $[35,36]$. When people diagnosed as AL, the potential unhealthy consequences, uncertain prognosis, and hopeless future do make it difficult for them to take positive coping behavior when confronted with the change of social status, the conflict of family roles, and the distress situation they struggling, which may lead to social discrimination, self-isolation, exacerbating internalized stigma. Contrary to acceptance-resignation, those taking confrontation showed more positive involvement in treatment compliance, information seeking and emotional self-adjustment without neglecting their physical or psychological health defects[24], suggesting positive coping strategy can be used as protective factor of stigma.

\subsubsection{Anxiety and depression}

The strong positive correlation between stigma and depression in cancer group was already demonstrated in many studies and depressed individuals were 2 times more likely to experience stigma[20,37]. In our study, $80.84 \%$ of AL patients accompany with anxiety while depression occurred in $83.83 \%$ of them. Moreover, $70.06 \%$ of them experienced two emotions above at the same time. Similar to previous researches, both of them were positively associated with stigma. But only depression was proved to be an influencing factor of stigma while anxiety was not entered into the linear regression. which is consistent with a meta-analysis[38], indicating that a higher level of stigma was observed among patients with high depression. Patients with depressive symptoms may be particularly vulnerable to inferiority complex, worthlessness, fear of being ridiculed and isolated, etc. These emotions would further strengthen the stigma, making them repulsive to seek medical treatment or reluctance to interact with others, resulting in condition aggravation and treatment delays.

\subsubsection{Demographic and clinical characteristics}

In addition to the impact of coping strategies and depression, patients' gender, fertility circumstance, family monthly income, degree of disease dependence and combined with chronic diseases were 
influencing factors of stigma, reminding us to pay close attention to how those characteristics as risk predictors contributing to stigma.

Gender influences feelings of social isolation, social reject, financial insecurity and internalized shame[11]. Higher stigma experiencing was found in male patients than female, particularly in youth aged $18 \sim 44$. That results were consistent with previous qualitative[39], quantitative studies[25, 40] or systematic review[13]. Male individuals, under the age of $18 \sim 44$ was actively involved in their life. When diagnosed with AL, many of them have to quit jobs and suffer a loss of family role or social status. They were in a large part labeled an undesirable personal trait. Being apart from routine life what it used to be dose yield pessimism and interpersonal relationship predicaments, making them vulnerable to stigma.

The findings suggested fertility should be consider as an important variable contributing to stigma which had not been reported before. Childless patients in our study undergoing stronger stigma compare to those who already finished fertility. Moreover, they were at high level in internalized shame dimension, with the average of item score $(3.1 \pm 0.504)$. However, no association was found between marital status and stigma level in regression analysis which was inconsistent with prior studies[20, 27]. In China's traditional conception of fertility, children are life renewing itself and deem to extensions of parents. A healthy body was an inevitably necessary requirement in continuation of the family line. After diagnosed with AL, fertility is basically deprived. Affected by such unacceptable reality and sociocultural stereotypes, patients might unable to complete psychological self-reconstruction.

Family per capita monthly income was demonstrated to be an influencing factor too, which was consistent with the results of Yilmaz's study[28] but contrary to Meyer's[41]. Depending on Yilmaz's view, low-income level negatively affected cancer-related stigma. The cost of AL treatment is substantial and increasing[32]. Higher income to some extent means lesser pressure to burden expenditure caused by illness. Emerging evidence[10] shows that stigma should be considered alongside the social determinants, such as socioeconomic status, social relationships. The existing economic conditions affect AL patients' attitude towards diseases and self [42], which may subsequently influence their perception of stigma.

Combined with chronical disease and Increased dependence expose advanced age people to more discrimination[28]. Older patients combined with chronic diseases had been shown to face double stigmatization[10] and display more negative attitudes[20]. Similar findings were confirmed in our study. Those two factors are relevant to severity of the illness of the patients, which is also consistent with previous findings that perceived severity of the illness, or a decrease in functional health status, did result in greater stigmatization[43]. This may due to at least two reasons. Firstly, the more severe illness, the greater dependence, the less able the individuals are to participate in limited activities of daily living. They may generally need caregivers to help them eat, dress even defecate on bed, strengthening their sense of self-shame. Secondly, chronic diseases affected the efficacy and prognosis of the therapy and one of its typical influence is to prolong the length of hospital stays. Therefore, these two factors may indirectly affect stigma experiencing via deteriorating functional health status. 


\section{Study Limitations}

The study has several limitations. The objects of this study are mainly concentrated in same area. The stigma and psychological states of AL patients in other provinces remain unknown. Besides, considering stigma is a sensitive topic, although we have ensured confidentiality of all information, a few patients might conceal their true feelings or even reported opposite answer, causing bias in results. Thus, it is suggested to expand sample size to minimize bias so as to confirm these findings in the future.

\section{Clinical Implications}

Stigma is a so common unhealthy psychological state existing in patients with cancer that it is often overlooked. Our study was primarily to identify the level of stigma and its influencing factors among patients with initial diagnosis of AL in China. Induced remission chemotherapy after diagnosed is deem to an optimal and crucial stage of $A L$ treatment and determines the success or failure of survival. Our findings emphasized it's equally important for medical staff and caregivers to not merely be focus on induction therapy but also help to relief stigma the patients undergoing when striving for complete remission. Moreover, close attention should be considered for those with following characteristics: male, childless, combine with chronic disease, exposed to financial crisis or high dependence. For those who exposed to risk factors of stigma, further effective interventions are suggested to alleviate patients' depression as well as to guide them to manage diseases with more positive coping strategies, thus protect them against stigma via these protective factors.

\section{Declarations}

\section{Funding information}

This study was supported by the Scientific Research Talents Training Project from Fujian Province Health Commission (Grant No. 2018-ZQN-63) and the Natural Scientific Foundation Project of Fujian Province (Grant No. 2019J01684).

\section{Conflict of Interest statement for all authors}

The authors declare no conflict of interest. All authors approved the final manuscript and have participated sufficiently in the work to take the responsibility for appropriate portions of the content.

\section{Availability of data and material}

The data that support the findings of this study are available from the corresponding author upon reasonable request.

\section{Code availability}

Not applicable. 


\section{Author contributions}

Rong $\mathrm{Hu}$, and Xiao-Ying Jiang contributed to the study conception and design.Miaoran-Lin, Ying-Chun Lin and Yi-mei Weng were responsible for Material preparation. Data collection and analysis were performed by Miao-Ran Lin. The first draft of the manuscript was written by Miao-Ran Lin and all authors commented on previous versions of the manuscript. All authors read and approved the final manuscript.

\section{Ethical approval}

The study was performed in accordance with the Declaration of Helsinki and was approved by the Ethics Committee of the Fujian Medical University (IRB Ref No: 2017/00049).

\section{Consent to participate}

Informed consent was obtained from all individual participants included in the study.

\section{Consent for publication}

Patients signed informed consent regarding publishing their data.

\section{References}

1. Ferrara F, Schiffer CA (2013) Acute myeloid leukaemia in adults. The Lancet 381:484-495

2. Bray F, Ferlay J, Soerjomataram I, Siegel RL, Torre LA, Jemal A (2018) Global cancer statistics 2018: GLOBOCAN estimates of incidence and mortality worldwide for 36 cancers in 185 countries Cacancer. J Clin 68:394-424

3. Chen W, Zheng R, Baade PD, Zhang S, Zeng H, Bray F, Jemal A, Yu XQ, He J (2016) Cancer Statistics in China, 2015 Ca-cancer J Clin 66: 115-132

4. Timilshina N, Breunis H, Tomlinson GA, Brandwein JM, Buckstein R, Durbano S, Alibhai SMH (2019) Long-term recovery of quality of life and physical function over three years in adult survivors of acute myeloid leukemia after intensive chemotherapy Leukemia 33: 15-25

5. Efficace F, Mandelli F, Platzbecker U, Cottone F, Lo Coco F (2015) Time to improve health-related quality of life outcomes in patients with acute promyelocytic leukemia Blood 126: 2523-2524

6. Tremolada M, Taverna L, Bonichini S, Basso G, Pillon M (2017) Self-Esteem and Academic Difficulties in Preadolescents and Adolescents Healed from Paediatric Leukaemia Cancers (Basel) 9

7. Buckley SA, Jimenez-Sahagun D, Othus M, Walter RB, Lee SJ (2018) Quality of life from the perspective of the patient with acute. myeloid leukemia Cancer 124:145-152

8. Goffman sociologyEJajo (1963) Stigma: Notes on the Management of Spoiled Identity

9. Major B, O'Brien LT (2005) The social psychology of stigma. Annu Rev Psychol 56:393-421

10. Hatzenbuehler ML, Phelan JC, Link BG (2013) Stigma as a Fundamental Cause of Population Health Inequalities. Am J Public Health 103:813-821 
11. Fife BL, Wright ER (2000) The dimensionality of stigma: a comparison of its impact on the self of persons with HIV/AIDS and cancer. J Health Soc Behav 41:50-67

12. Mascayano F, Armijo JE, Yang LH (2015) Addressing Stigma Relating to Mental Illness in Low- and Middle-Income Countries Front Psychiatry 6

13. Clement S, Schauman O, Graham T, Maggioni F, Evans-Lacko S, Bezborodovs N, Morgan C, Rusch N, Brown JS, Thornicroft G (2015) What is the impact of mental health-related stigma on help-seeking? A systematic review of quantitative and qualitative studies. Psychol Med 45:11-27

14. Xu Z, Ruesh N, Huang F, Koesters M (2017) Challenging mental health related stigma in China: Systematic review and meta-analysis. I Interventions among the general public Psychiat Res 255:449-456

15. Bosshard R, O'Reilly K, Ralston S, Chadda S, Cork D (2018) Systematic reviews of economic burden and health-related quality of life in patients with acute myeloid leukemia. Cancer Treat Rev 69:224232

16. Goss PE, Strasser-Weippl K, Chan A (2014) Challenges to effective cancer control in China, India, and Russia. Lancet Oncol 15:489-538

17. Ding T, Wang X, Fu A, Xu L, Lin J (2019) Anxiety and depression predict unfavorable survival in acute myeloid leukemia patients Medicine 98

18. Niedzwiedz CL, Knifton L, Robb KA, Katikireddi SV, Smith DJ (2019) Depression and anxiety among people living with and beyond cancer: a growing clinical and research priority. BMC Cancer 19:943

19. Gheihman G, Zimmermann C, Deckert A, Fitzgerald P, Mischitelle A, Rydall A, Schimmer A, Gagliese L, Lo C, Rodin G (2016) Depression and hopelessness in patients with acute leukemia: the psychological impact of an acute and life-threatening disorder Psycho-oncol 25: 979-989

20. Cho J, Choi E-k, Kim SY, Shin DW, Cho B-L, Kim C-h, Koh DH, Guallar E, Bardwell WA, Park JH (2013) Association between cancer stigma and depression among cancer survivors: a nationwide survey in Korea Psycho-oncol 22: 2372-2378

21. Greer JA, Jacobs JM, El-Jawahri A, Nipp RD, Gallagher ER, Pirl WF, Park ER, Muzikansky A, Jacobsen JC, Jackson VA, Temel JS (2018) Role of Patient Coping Strategies in Understanding the Effects of Early Palliative Care on Quality of Life and Mood. J Clin Oncol 36:53-60

22. Greer JA, Applebaum AJ, Jacobsen JC, Temel JS, Jackson VA (2020) Understanding and Addressing the Role of Coping in Palliative Care for Patients With Advanced Cancer. Journal of clinical oncology: official journal of the American Society of Clinical Oncology 38:915-925

23. Ghodraty-Jabloo V, Alibhai SMH, Breunis H, Puts MTE (2016) Keep your mind off negative things: coping with long-term effects of acute myeloid leukemia (AML). Support Care Cancer 24:2035-2045

24. Zhang J-e, Yuan J-m (2018) Stigma and its influencing factors among Chinese patients with stoma Psycho-oncol 27: 174-175

25. Liu XH, Zhong JD, Zhang JE, Cheng Y, Bu XQ (2020) Stigma and its correlates in people living with lung cancer: A cross-sectional study from China Psycho-oncol 29: 287-293 
26. Barroso J, Relf MV, Williams MS, Arscott J, Moore ED, Caiola C, Silva SG (2014) A randomized controlled trial of the efficacy of a stigma reduction intervention for HIV-infected women in the Deep South AIDS Patient Care. STDS 28:489-498

27. Ernst J, Mehnert A, Dietz A, Hornemann B, Esser P (2017) Perceived stigmatization and its impact on quality of life - results from a large register-based study including breast, colon, prostate and lung cancer patients BMC Cancer 17

28. Yilmaz M, Dissiz G, Usluoglu AK, Iriz S, Demir F, Alacacioglu A (2020) Cancer-Related Stigma and Depression in Cancer Patients in A Middle-Income Country Asia-Pacific. journal of oncology nursing 7:95-102

29. Pan A-W, Chung L, Fife BL, Hsiung P-C (2007) Evaluation of the psychometrics of the Social Impact Scale: a measure of stigmatization. International journal of rehabilitation research Internationale Zeitschrift fur Rehabilitationsforschung Revue internationale de recherches de readaptation 30:235238

30. Sun Z, HX L, LY J, LN Y TZ, F J (2017) The reliability and validity of hospital Anxiety and Depression Scale Chinese. Journal of Clinicians 11:198-201

31. Shen X, G J (2000) Chinese version of medical coping Style questionnaire: A test report of 701 cases. Chinese hehavioral medical science: $22-24$

32. Carrera PM, Kantarjian HM, Blinder VS (2018) The Financial Burden and Distress of Patients with Cancer: Understanding and Stepping-Up Action on the Financial Toxicity of Cancer Treatment Ca-. cancer J Clin 68:153-165

33. Rinehart R, Rao D, Amico RK, Ruiz E, Brandes P, Correa C, Pasalar S, Lama JR, Duerr A, Molina Y (2019) Experienced HIV-Related Stigma and Psychological Distress in Peruvian Sexual and Gender Minorities: A Longitudinal Study to Explore Mediating Roles of Internalized HIV-Related Stigma and Coping Styles. AIDS Behav 23:661-674

34. Xia Z (2017) Effects of psychological intervention on coping style, psychological state and quality of life of leukemia patients undergoing chemotherapy. Medical Theory Practice 30:3743-3745

35. Chen S, Mei R, Tan C, Li X, Zhong C, Ye M (2020) Psychological resilience and related influencing factors in postoperative non-small cell lung cancer patients: A cross-sectional study.

Psychooncology 29:1815-1822

36. Feifel H, Strack S, Nagy VT (1987) Coping strategies and associated features of medically ill patients. Psychosom Med 49:616-625

37. Schroyen S, Marquet M, Jerusalem G, Dardenne B, Van den Akker M, Buntinx F, Adam S, Missotten P (2017) The link between self-perceptions of aging, cancer view and physical and mental health of older people with cancer: A cross-sectional study. J Geriatr Oncol 8:64-68

38. Shi Y, Wang S, Ying J, Zhang M, Liu P, Zhang H, Sun J (2017) Correlates of perceived stigma for people living with epilepsy: A meta-analysis. Epilepsy Behav 70:198-203

39. Liu H, Yang Q, Narsavage GL, Yang C, Chen Y, Xu G, Wu X (2016) Coping with stigma: the experiences of Chinese patients living with lung cancer Springerplus 5 
40. Qin F, Ye X, Wei H, Wen Y, Shi L, Zhen L, Zhu M, Zhang L (2019) Sexual Experience and Stigma Among Chinese Patients With an Enterostomy: A Cross-sectional. Descriptive Study Wound Management Prevention 65:22-30

41. Meyer IH, Brown TNT, Herman JL, Reisner SL, Bockting WO (2017) Demographic Characteristics and Health Status of Transgender Adults in Select US Regions: Behavioral Risk Factor Surveillance System, 2014. Am J Public Health 107:582-589

42. Pandya BJ, Chen CC, Medeiros BC, McGuiness CB, Wilson S, Horvath Walsh LE, Wade RL (2019) Economic and Clinical Burden of Relapsed and/or Refractory Active Treatment Episodes in Patients with Acute Myeloid Leukemia (AML) in the USA: A Retrospective Analysis of a Commercial Payer. Database Adv Ther 36:1922-1935

43. Dhopeshwarkar N, lqbal S, Wang X, Salas M (2019) A Retrospective Study of Comorbidities and Complications in Elderly Acute Myeloid Leukemia Patients in the United States Clinical Lymphoma Myeloma. \& Leukemia 19:E436-E456 